\title{
Structure Refinement of the Glucocorticoid Receptor-DNA Binding Domain from NMR Data by Relaxation Matrix Calculations
}

\author{
Marc A. A. van Tilborg', Alexandre M. J. J. Bonvinn, ${ }^{1,2}$ Karl Hård' \\ Adrian L. Davis ${ }^{1,3}$, Bonnie Maler ${ }^{4}$, Rolf Boelens', Keith R. Yamamoto ${ }^{4}$ \\ and Robert Kaptein ${ }^{1 *}$
}

'Bijuoet Center for

Biomolecular Research

Utrecht University, Padualaan

8, NL-3584 CH Utrecht

The Netherlands

${ }^{2}$ Present address: Department of Molecular Biophysics and Biochemistry, Yale University P.O. Box 6666, New Haven CT 06511, U.S.A.

${ }^{3}$ Present Address: Shell

Research Thornton

P.O. Box 1, Chester

Cheshire CH1 3SH, U.K.

${ }^{4}$ Departments of

Pharmacology, and

Biochemistry and Biophysics

University of California, San

Francisco, CA 94143-0450

U.S.A.
The solution structure of the glucocorticoid receptor (GR) DNA-binding domain (DBD), consisting of 93 residues, has been refined from two and three-dimensional NMR data using an ensemble iterative relaxation matrix approach followed by direct NOE refinement with DINOSAUR. A set of 47 structures of the rat GR fragment Cys440-Arg510 was generated with distance geometry and further refined with a combination of restrained energy minimization and restrained molecular dynamics in a parallel refinement protocol. Distance constraints were obtained from an extensive set of NOE build-up curves in $\mathrm{H}_{2} \mathrm{O}$ and ${ }^{2} \mathrm{H}_{2} \mathrm{O}$ via relaxation matrix calculations (1186 distance constraints from NOE intensities, $10 \phi$ and $22 \chi_{1}$ dihedral angle constraints from $J$ - coupling data were used for the calculations). The root-mean-square deviation values of the 11 best structures on the well-determined part of the protein (Cys440 to Ser448, His 451 to Glu469 and Pro 493 to Glu508) are $0.60 \AA$ and $1.20 \AA$ from the average for backbone and all heavy atoms, respectively. The final structures have $R$-factors around 0.40 and good stereochemical qualities.

The first zinc-coordinating domain of the GR DBD is very similar to the crystal structure with a root-mean-square difference of $1.4 \AA$. The second zinc-coordinating domain is still disordered in solution. No secondary structure element is found in this domain in the free state. As suggested by crystallographic studies on the estrogen receptor DBD-DNA and GR DBDDNA complexes, part of this region will form a distorted helix and the D-box will undergo a conformational change upon cooperative binding to DNA.

Keywords: glucocorticoid receptor; DNA binding domain; zinc finger direct NOE refinement; solution structure

\section{Introduction}

The glucocorticoid receptor (GR), like all other members of the steroid/thyroid receptor family, is

Abbreviations used: COSY, correlated spectroscopy; DBD, DNA-binding domain; DGII, distance geometry; DINOSAUR, direct NOE simulation approach for unbelievable structure refinement; EM, energy minimization; ER, estrogen receptor; GR, glucocorticoid receptor; HSQC, heteronuclear single quantum coherence; IRMA, iterative relaxation matrix approach; MD, molecular dynamics; NOESY, nuclear Overhauser enhancement spectroscopy; RAR, retinoic acid receptor $\beta ;$ REM, restrained energy minimization; $R M D$, restrained molecular dynamics; $R X R$, retinoic $x$ receptor; TOCSY, total correlation spectroscopy; TPPI, timeproportional phase increment; $2 \mathrm{D}$ and $3 \mathrm{D}$, two and three-dimensional; r.m.s.d., root-mean-square deviation. structurally organized into three domains (Evans, 1988; Ham \& Parker, 1989) related to the function of the protein as a ligand-dependent transcription factor. The A/B domain comprises the $\mathrm{N}$-terminal domain and participates in protein-protein interactions. The $\mathrm{C}$ domain or DNA-binding domain (DBD) is involved in protein-DNA interactions. Finally, the E/F domain binds small hydrophobic ligands, e.g. glucocorticoid hormone.

The structure of the GR DBD was determined in solution, using NMR spectroscopy and distance geometry calculations by Härd et al. $(1990 a, b)$. Several secondary structure elements within fragments KPARP[Cys440-Gly525]RL have been identified (Figure 1). These elements include two $\alpha$-helical regions encompassing Gly458 to Glu469 and Pro493 to Ala503, and a short stretch of anti-parallel $\beta$-sheet involving residues Cys440 to 


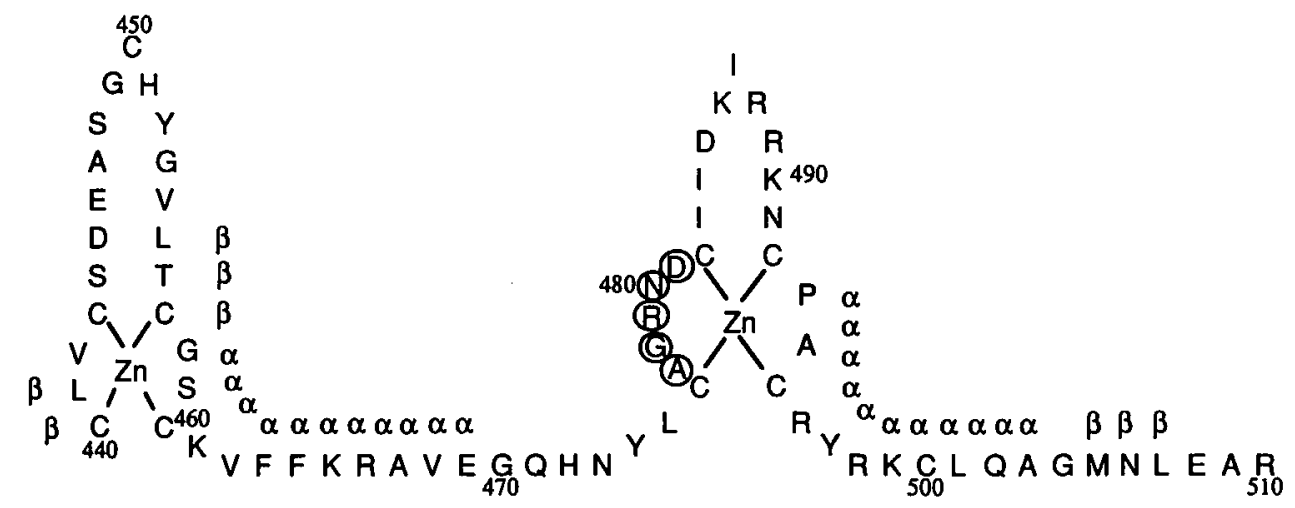

Figure 1. The sequence and zinc-coordination of the glucocorticoid receptor (GR) DNA-binding domain (Cys440 to Arg510); numbering refers to the rat GR. The helical segments of the solution structure are indicated by $\alpha$, the residues in the D-box are circled and $\beta$-sheet regions are indicated by $\beta$.

Leu441 and Leu455 to Cys457. The first helix is followed by an extended region and a short loop, the so called D-box (Ala477 to Asp481). The GR DBD contains nine cysteine residues, of which eight coordinate two zinc ions in a tetrahedral fashion. A crystallographic analysis of the interaction of GR DBD with DNA was reported by Luisi et al. (1991). The protein binds its DNA target site as a dimer. The overall structure of the protein in the complex is similar to that in the free state, but there are differences in the second zinc-coordinating domain. A short distorted helix (Lys486 to Asn491) was observed in the co-crystal DNA complex, whereas in the NMR structure of free GR DBD this fragment was disordered. It was proposed that the differences between the NMR and crystal structure might arise from stabilization of the structure upon DNA binding. NMR studies of the estrogen receptor (ER) DBD (Schwabe et al., 1990, 1993a), retinoic acid receptor $\beta$ (RAR) DBD (Knegtel et al., 1993) and retinoic $X$ receptor (RXR) DBD (Lee et al., 1993) also revealed structures that are very similar to that of the GR DBD, while no distorted helix is found in the second zinc finger of the unbound solution structure. Recently the structure of a ten residue shorter GR DBD fragment (M[Leu439Gln520]) has been expressed and refined by Baumann et al. (1993). In this fragment, a distorted helix (Lys486 to Asn491) was found similar to that observed in the X-ray structure of the complex (Luisi et al., 1991). This raises the question of whether DNA binding is accompanied by relatively large conformational changes in the second finger region, as suggested by our earlier work, or whether the distorted helix involved in proteinprotein interactions is partially preformed in the free protein. This question is addressed here, where we present the refined solution structure of GR DBD (Cys440 to Arg510) from 2D and 3D NMR data, obtained from relaxation matrix calculations. In the following, this structure is referred to as DBD93, whereas the structure reported by Baumann et al. (1993) is termed DBD82. First, accurate distance constraints obtained from the iterative relaxation matrix approach (IRMA: Boelens et al., 1988, 1989; Koning, 1990; Koning et al., 1990, 1991) are used to refine a set of structures. The resulting structures are subsequently refined with the direct use of the experimental NOE intensities as constraints following a slow-cooling simulated annealing protocol using the DINOSAUR program (Bonvin et al., 1993). Finally, the refined solution structure of GR $\mathrm{DBD}$ is analyzed in detail and compared with the structure of GR DBD in the co-crystal DNA complex (Luisi et al., 1991) and the refined DBD82 (Baumann et al., 1993).

The overall folding of the protein is the same in the refined structures and the co-crystal DNA complex but, in contrast to Baumann et al. (1993), parts of the second zinc-coordination domain (residues Ala477 to Asp481 and Lys486 to Asn491) are still disordered in the solution structure. Implications for the mode of DNA binding by GR are discussed.

\section{Results and Discussion}

\section{Generalized order parameter $\boldsymbol{S}^{2}$}

From a $55 \mathrm{ps} \mathrm{MD}$ trajector all interproton vectors within $5 \AA$ were calculated, resulting in a set of 5521 internal correlation functions. As observed by Bonvin et al. (1993), a correlation can be found between computed generalized order parameters and the secondary structure. In Figure 2 this is illustrated by a plot of the intraresidue $\mathrm{H}^{\mathrm{N}}-\mathrm{H}^{a}$ order parameters as a function of the residue number. From this Figure it can be seen that the $\alpha$-helical regions $\alpha_{1}$ and $\alpha_{2}$ have higher $S^{2}$ values than other parts of the protein. The region where the distorted helix and the D-box are found in the X-ray structure (Luisi et al., 1991) show in our case, on average, more mobility than the two helical regions. 


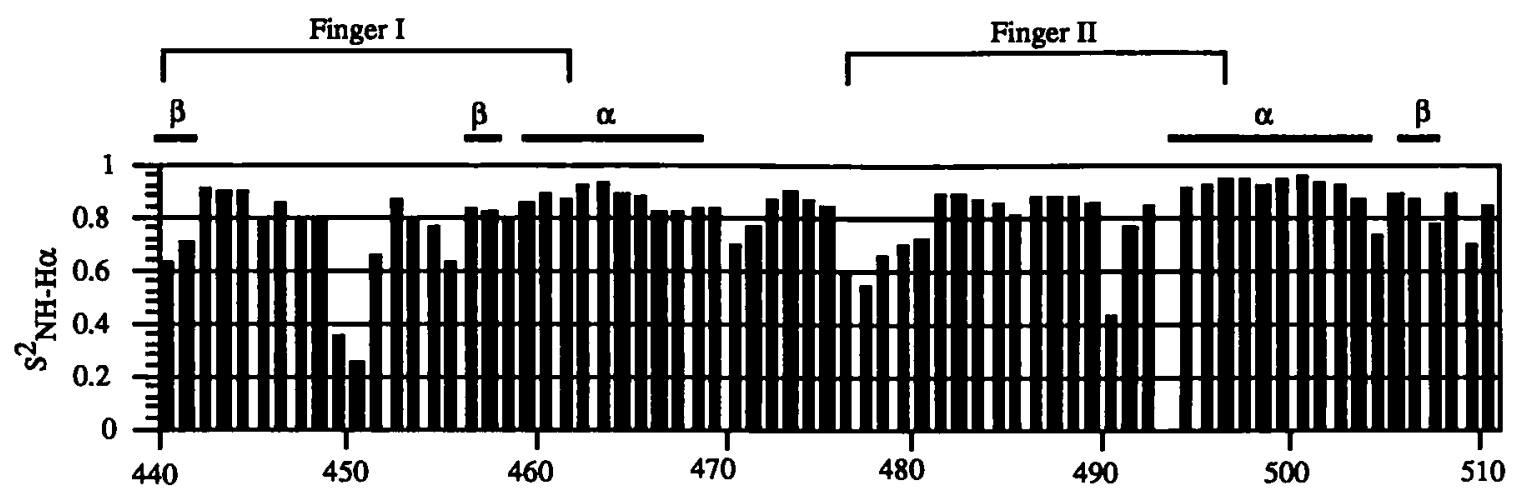

Figure 2. Calculated generalized order parameters $S_{\mathrm{N}-\mathrm{H}}^{2}$ as a function of the residue number of the final $11 \mathrm{GR}$ DBD structures.

\section{Structure refinement}

A view of the superimposed backbone traces of the 11 best GR DBD structures during the refinement is presented in Figure 3. This Figure shows the improvement of the 11 structures during refinement. In Table 1 the course of the refinement can be seen in terms of energy and $R$-factor. The distance restraint energy of the first four stages was calculated for the set of 1186 distance constraints. The distribution of these constraints as a function of the residue number is illustrated in Figure 4(a). The average constraint violation after this stage of the refinement is $0.02 \AA$ with a total number of distance violations of 183 . None exceeds $1.0 \AA$, and ten are larger than $0.7 \AA$. The deviation from the ideal covalent geometry was $0.02 \AA$ for the bond length and $3.4^{\circ}$ for the bond angle.
The distance restraint energy decreased during the IRMA refinement (refinement against distances). The NOE energy remained the same during this stage but decreased drastically after direct NOE refinement. Small differences in $R$-factor and $Q^{1 / 6}$ factor (Table 1) were found between the subsequent IRMA cycles, indicating that convergence was reached after the third IRMA cycle. The direct NOE refinement resulted in a further decrease in $R$ and $Q^{1 / 6}$ factors. Figure $4(\mathrm{~b})$ shows the $Q^{1 / 6}$ factor for the residues, most with low values around 0.04 , indicating that the final structures fit the experimental data very well.

In Table 2, r.m.s.d. values of specific parts of the GR DBD fragment in different stages of the refinement are presented. This Table shows that the r.m.s.d. values decreased during IRMA refinement and remain virtually the same in the last step of the

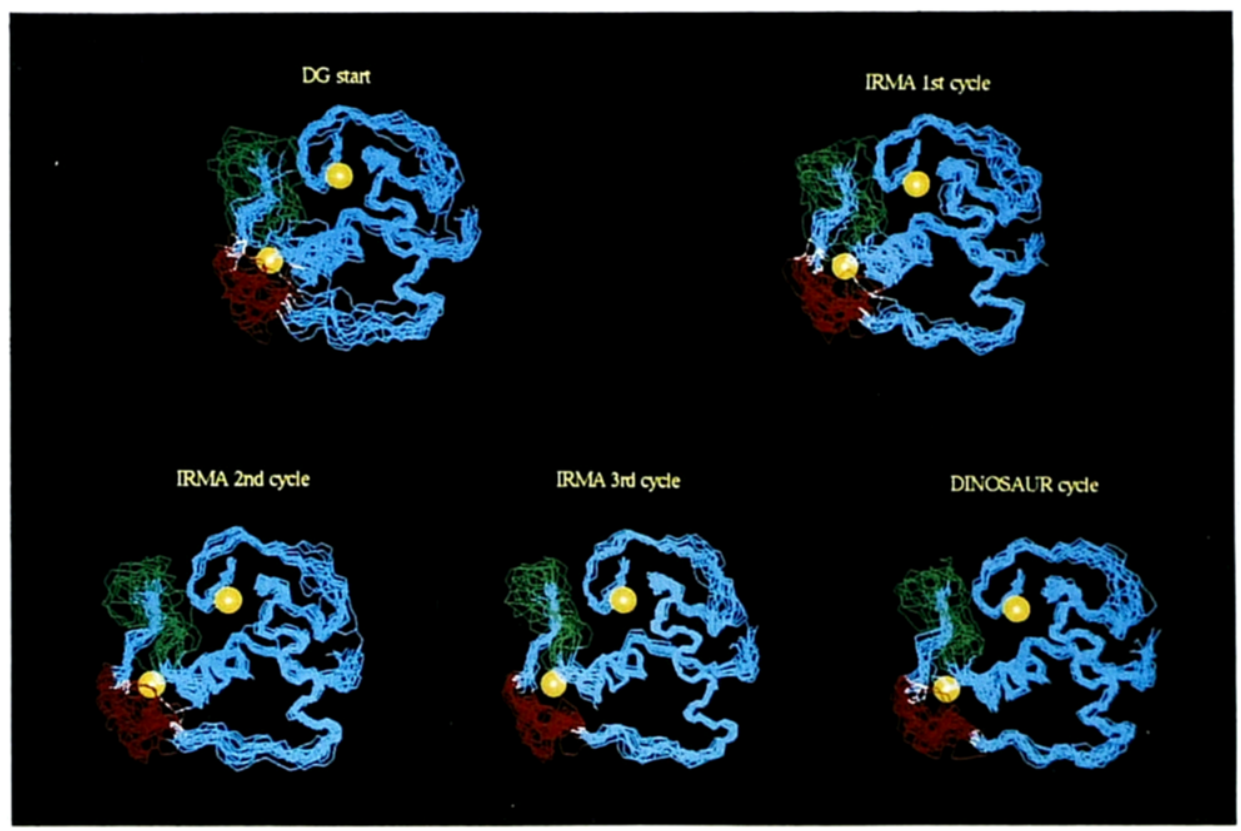

Figure 3. Overlay of the 11 structures of the GR DBD during the refinement stages. Structures are shown after DG/EM, the first, second and third IRMA cycle and after the last stage of the refinement. The structures are superimposed on $\mathrm{C}^{\alpha}$, $\mathrm{C}$ and N atoms of the well-defined regions of GR DBD (Cys440 to Ser448, His451 to Glu469 and Pro493 to Glu508). The problem regions, the D-box and the distorted region in the second finger, are colored red and green, respectively. 


\section{Table 1}

Course of the refinement in terms of energies (in $\mathrm{kJ} \mathrm{mol}^{-1}$ ) and $R$-factors of the $11 \mathrm{GR}-\mathrm{DBD}$ structures

\begin{tabular}{|c|c|c|c|c|c|}
\hline & Start DG/REM & IRMA-1 & IRMA-2 & IRMA-3 & DINOSAUR \\
\hline \multicolumn{6}{|l|}{ Energy } \\
\hline Epot & $-4721 \pm 213$ & $-4250 \pm 235$ & $-4797 \pm 152$ & $-4856 \pm 70$ & $-2842 \pm 211$ \\
\hline Edisre $^{b}$ & $2697 \pm 643$ & $1660 \pm 252$ & $1201 \pm 172$ & $623 \pm 67$ & \\
\hline Edihre $^{r}$ & $453 \pm 148$ & $474 \pm 91$ & $210 \pm 142$ & $105 \pm 67$ & $146 \pm 62$ \\
\hline $\begin{array}{l}\text { Enoed } \\
R \text {-factor }\end{array}$ & $16255 \pm 958$ & $15982 \pm 1276$ & $15072 \pm 1272$ & $15092 \pm 1474$ & $7415 \pm 305$ \\
\hline$R$ & $0.61 \pm 0.01$ & $0.58 \pm 0.01$ & $0.55 \pm 0.01$ & $0.57 \pm 0.01$ & $0.42 \pm 0.01$ \\
\hline Rens & 0.56 & 0.54 & $0 . \overline{5} 2$ & 0.54 & 0.40 \\
\hline$Q^{1 / \hbar}$ & $0.151 \pm 0.007$ & $0.139 \pm 0.004$ & $0.129 \pm 0.004$ & $0.131 \pm 0.003$ & $0.098 \pm 0.004$ \\
\hline$Q^{1 / 4 x^{\prime \prime 1 s}}$ & 0.128 & $0 . \overline{1} 22$ & $0 . \overline{1} 20$ & 0.120 & 0.084 \\
\hline
\end{tabular}

-GROMOS force field, restraint excluded.

' Harmonic potential (Kaptein ef al., 1985), force constant $=40 \mathrm{~kJ} \mathrm{~mol}^{-1} \AA^{-2}$. The energy was calculated for the set of 1186 distance constraints obtained in the IRMA-3 cycle.

'Dihedral angle restraint potential $\left(V_{\text {dihre }}=K_{\text {dilure }}\left[1-\cos \left(\phi-\phi_{0}\right)\right]\right.$, de Vlieg et al., 1986), force constant $=110 \mathrm{~kJ} \mathrm{~mol}^{-1}$.

$\checkmark$ NOE potential as proposed by Bonvin et al. (1994b) with a force constant of $400 \mathrm{~kJ} \mathrm{~mol}^{-1}$. The weighting factors $i 0$, were calculated with the experimental noise level $(N)$ and a relative error $\epsilon=5 \%$.

- Average and ensemble $R$-factors calculated for a set of 11 structures using all NOE peaks at 3 mixing times. The ensemble $R$-factors were calculated from an average relaxation matrix.

$$
\begin{aligned}
& R=\frac{\sum_{i} \tau_{m}\left|f A_{i}^{t(m) n}-A_{i}^{m p}\right|}{\sum \tau_{m}\left|A_{i}^{u p p}\right|}
\end{aligned}
$$

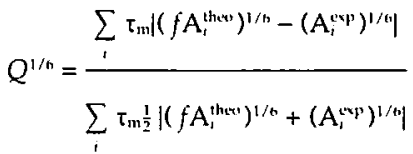

refinement. All r.m.s.d. values were calculated with respect to the averaged structure unless indicated otherwise. Superposition of the bestdetermined part of the molecule (residues Cys440 to Ser448, His451 to Glu469 and Pro493 to Glu508) resulted in a r.m.s.d. of $0.60 \AA$. The positions of helix 1 and 2 relative to each other (superposition on helix 1 and helix 2) results in a r.m.s.d. of $0.36 \AA$. The structure of the first domain (Cys 440 to Gly470) is much better defined than that of the second domain ( $G \ln 471$ to Arg510) with a backbone r.m.s.d. of $0.68 \AA$ and $1.72 \AA$, respectively with respect to the average. From Figure 4(c) and (d) three distinct problem regions in the structure can be identified. Some disorder in the tip of the first zinc-coordinating domain (Ser448 to His451) is reflected in somewhat higher $C^{x}$ and all-atom r.m.s.d. values and low $S^{\phi}$ and $S^{{ }^{\prime}}$ parameters (Figure 4(e) and $(f)$ ). Much less ordered are the D-box (Ala477 to Asp481) and the fragment Lys486 to Asn491, both in the second zinc-coordinating domain. Inspection of Figure 4(c) and (d) reveals that these parts of the molecule have both high $\mathrm{C}^{\alpha}$ and all-atom r.m.s.d. values, in combination with the low angular order parameter values for the backbone dihedral angles, indicating a less well defined substructure.

\section{Analysis of the structure}

The secondary structure elements of GR DBD were assigned according to the criteria of Kabsch \& Sander (1983). In the first zinc-coordinating domain, the residues Cys440-Leu441 and Leu 455 to Cys 457 form a short stretch of anti-parallel $\beta$-sheet with a hydrogen bond between the amide group of Leu441 and the carbonyl group of Leu455. The $\phi$ and $\Psi$ angles are around $-100^{\circ}$ and $-120^{\circ}$, respectively, and are well defined, having high angular order parameters (Figure 4(e) and (f)). The $\beta$-sheet Leu 455 to Cys 457 is followed by an $\alpha$-helix from Gly 458 to Glu 469 with typical $\phi$ and $\Psi$ angles around $-57^{\circ}$ and $-59^{\circ}$, respectively. The typical pattern of hydrogen bonds involving amide protons and carbonyl groups in the $\alpha$-helical region is found (data not shown). This first helix is linked to the second zinc-coordinating domain by an extended structure from $G \ln 471$ to Leu475. The D-box (Ala477 to Asp481), included in this second zinc-coordinating domain, is not as well defined as other parts of the GR DBD, which is reflected in angular order parameters around 0.7 . Following this D-box is a well-defined fragment Cys482 to Asp485, subsequently followed by a disordered fragment Lys486 to Asn491. Low angular order parameters and high $\mathrm{C}^{*}$ and all-atom r.m.s.d. values are shown in Figure $4(\mathrm{c})$ and (d). This disordered 


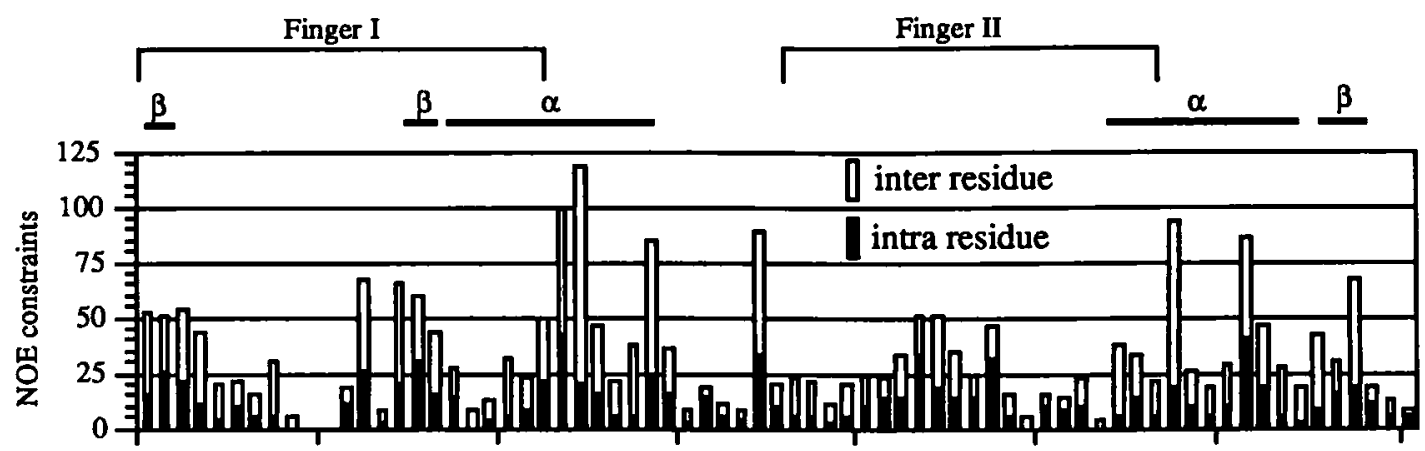

(a)

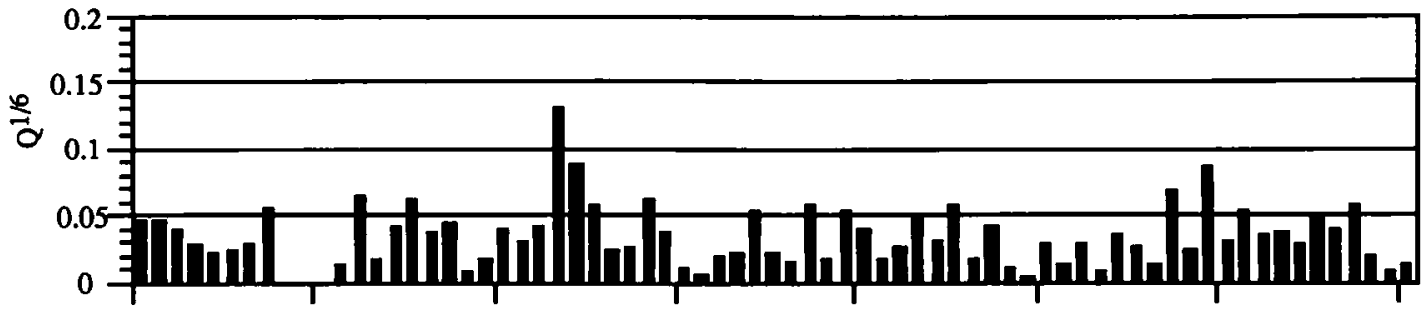

(b)
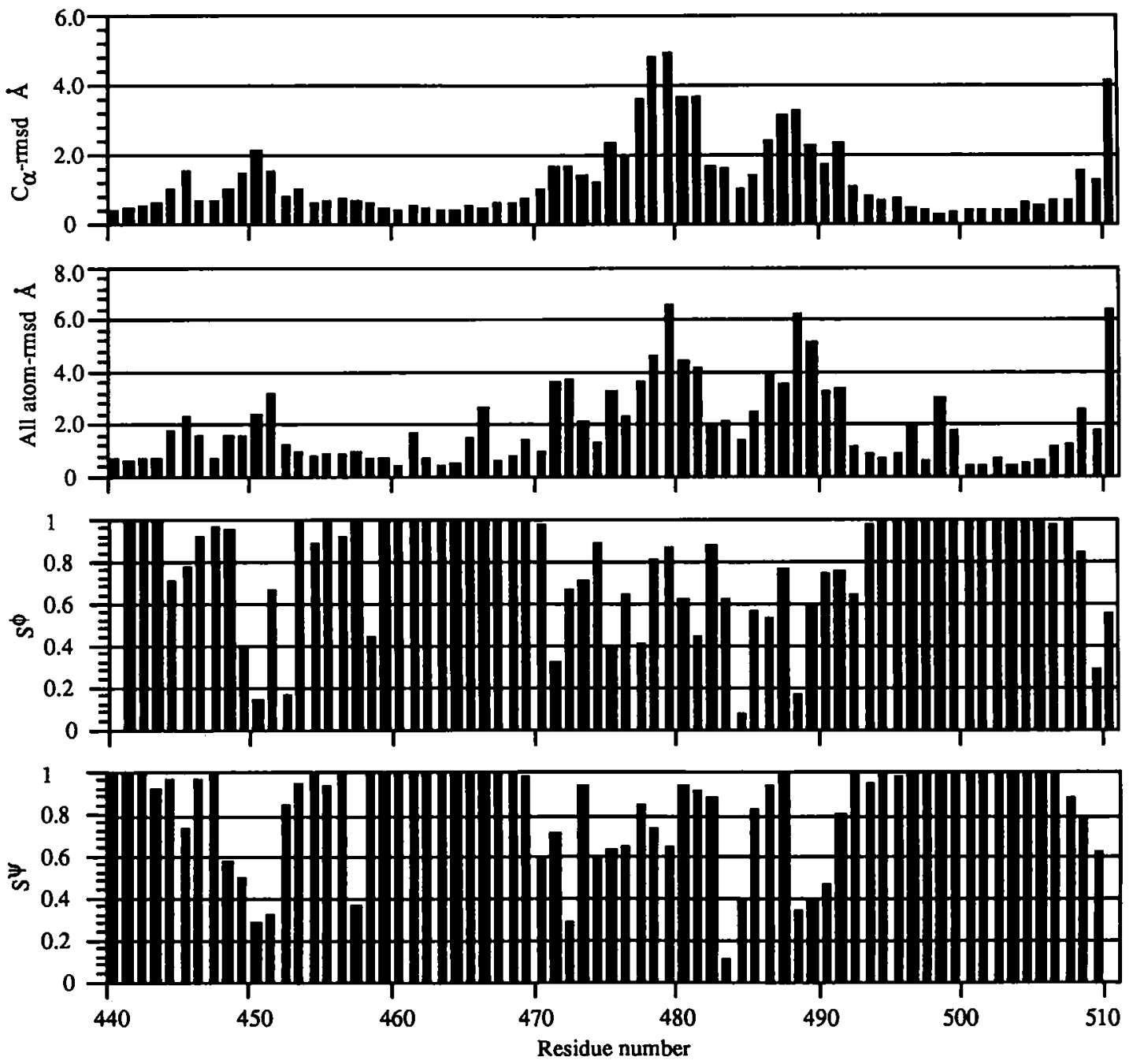

Figure 4. (a), Number of inter and intra-residue NOE constraints as a function of the residue number of GR DBD. (b), $Q^{1 / 6}$ factors as a function of the residue number of GR DBD. The r.m.s.d. from the average on $C^{x}$ atoms (c) and all-atoms r.m.s.d. (d), both for the well-defined region Cys440 to Ser448, His451 to Glu469 and Pro493 to Glu508. Backbone angular order parameters $S^{\Phi}(\mathrm{e})$ and $S^{\psi}(\mathrm{f})$ as function of the residue number of the final $11 \mathrm{GR}$ DBD structures. 
Table 2

\begin{tabular}{|c|c|c|c|c|c|c|}
\hline & $\begin{array}{c}\text { Start } \\
\text { DG/REM }\end{array}$ & IRMA-1 & IRMA-2 & IRMA-3 & DINOSAUR & $\begin{array}{c}\text { NMR versus } \\
\text { X-ray }\end{array}$ \\
\hline \multicolumn{7}{|l|}{ r.m.s.d. $(\AA)$} \\
\hline Best & a $1.56 \pm 0.34$ & $1.39 \pm 0.33$ & $1.30 \pm 0.28$ & $1.11 \pm 0.21$ & $1.20 \pm 0.18$ & \\
\hline determined & b $0.88 \pm 0.28$ & $0.67 \pm 0.26$ & $0.66 \pm 0.22$ & $0.54 \pm 0.15$ & $0.60 \pm 0.14$ & $1.41 \pm 0.10$ \\
\hline \multirow[t]{2}{*}{$\alpha^{\text {distorted }}$} & a $4.67 \pm 0.44$ & $4.47 \pm 0.90$ & $4.08 \pm 0.55$ & $3.80 \pm 0.37$ & $3.64 \pm 0.50$ & \\
\hline & b $1.27 \pm 0.23$ & $1.27 \pm 0.23$ & $1.30 \pm 0.17$ & $1.14 \pm 0.23$ & $1.16 \pm 0.21$ & $2.00 \pm 0.30$ \\
\hline \multirow[t]{2}{*}{$\alpha^{1}+\alpha^{2}$} & a $1.50 \pm 0.36$ & $1.31 \pm 0.24$ & $1.20 \pm 0.25$ & $1.05 \pm 0.20$ & $1.03 \pm 0.14$ & \\
\hline & b $0.75 \pm 0.34$ & $0.55 \pm 0.34$ & $0.49 \pm 0.30$ & $0.32 \pm 0.12$ & $0.36 \pm 0.10$ & $1.10 \pm 0.07$ \\
\hline \multirow[t]{2}{*}{ 1st domain } & a $1.32 \pm 0.19$ & $1.29 \pm 0.16$ & $1.27 \pm 0.19$ & $1.24 \pm 0.25$ & $1.21 \pm 0.26$ & \\
\hline & b $0.80 \pm 0.15$ & $0.73 \pm 0.19$ & $0.70 \pm 0.15$ & $0.69 \pm 0.16$ & $0.68 \pm 0.14$ & $1.34 \pm 0.13$ \\
\hline \multirow[t]{2}{*}{ 2nd domain } & a $3.36 \pm 0.32$ & $3.33 \pm 0.28$ & $3.00 \pm 0.44$ & $2.75 \pm 0.28$ & $2.79 \pm 0.26$ & \\
\hline & b $2.28 \pm 0.40$ & $2.19 \pm 0.37$ & $1.94 \pm 0.46$ & $1.63 \pm 0.25$ & $1.72 \pm 0.29$ & $3.00 \pm 0.35$ \\
\hline
\end{tabular}

The r.m.s.d. from the 11 structures with respect to the average structure for the region: Best determined, C440-S448,

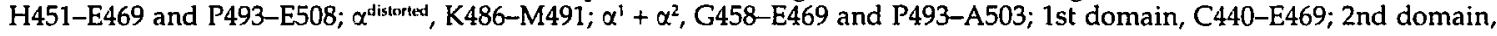
G470-R510; a, all atoms; b, backbone atoms.

part of the GR DBD fragment is followed by a second helical region (Pro493 to Ala503), which is characterized by high $S^{\phi}$ and $S^{\boldsymbol{\Psi}}$ values, and angles around $-60^{\circ}$. The usual pattern of hydrogen bonds for helices is observed in this second helical region. The two helices are oriented approximately perpendicular to each other and a hydrophobic core is formed between them, comprising five well-defined aromatic residues Tyr452, Phe463, Phe464, Tyr474 and Try497. Pro493, Cys500, Met505 and the hydrocarbon side-chain of Arg 496 are also part of the hydrophobic core, all surrounding the aromatic cluster. At the $C$ terminus there is a short strand of well-defined $\beta$-strand encompassing Met505 to Leu507. This $\beta$-strand was not observed in the initial structure (Härd et al., 1990a,b).

The stereochemical quality of the ensemble of 11 structures has been checked with the program PROCHECK (Morris et al., 1992) and the results are summarized in Table 3. Besides several glycine residues, on average there are only two amino acid residues, S444 and D481, found to have positive $\phi$ angles in the forbidden region of the Ramachandran plot.

The two zinc-coordinating domains differ in the chirality of the arrangement of zinc ligands. In the first zinc-coordinating domain the chiral zinc-sulfur

\section{Table 3}

Stereochemical quality of the ensemble of 11 NMR structures of the DINOSAUR refinement

\begin{tabular}{lcc}
\hline Parameters & Ideal values & \multicolumn{1}{c}{ GRDBD values } \\
\hline$\chi_{1}$ dihedral $g(-)$ & $64.1 \pm 15.7$ & $60.9 \pm 12.1$ \\
$\chi_{1}$ dihedral trans & $183.6 \pm 16.8$ & $184.7 \pm 19.7$ \\
$\chi_{1}$ dihedral $g(+)$ & $-66.7 \pm 15.0$ & $-69.4 \pm 23.6$ \\
$\chi_{2}$ dihedral & $177.4 \pm 18.5$ & $169.4 \pm 23.0$ \\
Proline $\phi$ & $-65.4 \pm 11.2$ & $-60.9 \pm 15.4$ \\
Helix $\phi$ & $-65.3 \pm 11.9$ & $-61.8 \pm 14.6$ \\
Helix $\Psi$ & $-39.4 \pm 11.3$ & $-44.7 \pm 18.1$ \\
$\omega$ dihedral & $180.0 \pm 5.8$ & $180.5 \pm 5.9$ \\
H-bond energy & $-2.0 \pm 0.8$ & $-1.8 \pm 0.8$ \\
Chirality $C^{\mathrm{s}}$ & $33.9 \pm 3.5$ & $30.3 \pm 5.8$ \\
\hline
\end{tabular}

"Ideal values according to Morris et al. (1992; PROCHECK).

b Calculated from the final set of structures with PROCHECK. center has an $S$ configuration while the second domain has an $\mathrm{R}$ configuration. Both configurations are in accordance with those found in the X-ray structures of the GR DBD and ER DBD complexes (Luisi et al., 1991; Schwabe et al., 1993b). We note that the chirality of the second finger in the present structure is different from that in the low-resolution structure reported by Härd (1990b).

\section{Solution structure versus X-ray structure}

We shall first compare the refined solution structure of GR DBD ( 440 to 510 ) with the structure of a monomer in the crystal structure of the

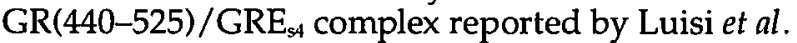
(1991). In this complex only one of the DBD monomers binds specifically to DNA, but the backbone r.m.s.d. value between the two monomers is $0.7 \AA$, indicating that they are very similar. The NMR $R$-factor and $Q^{1 / 6}$ factor computed for the crystal structure are close to the values obtained for the final IRMA structures (values for the $X$-ray structure are 0.51 and 0.134 , respectively). In Table 2, r.m.s.d. values between different parts of the refined solution structure and the crystal structure are summarized. Superposition of the average backbone (red) of residues (Cys440 to Ser448, His451 to Glu469, Pro493 to Glu508) from the 11 NMR structures with that of the protein in the crystal structure (blue), results in a r.m.s.d. value of $1.4 \AA$ (Figure 5). The relative orientation of the two helices in solution and crystal is also almost the same with a backbone r.m.s.d. of $1.10 \AA$.

No large structural difference is observed in the first domain (Cys440 to Gly470) between the backbone of the refined solution structure and the crystal structure (backbone r.m.s.d. around $1.34 \AA$, with the exception of the tip of this first finger (Gly449 to His451)), which is not. well defined in the solution structure. In the crystal structure, a well-defined hydrogen bond network has been found in the tip of the first zinc-containing finger (His451-Ser448-Gly458), which is not found in the present structure. As already indicated, the second 


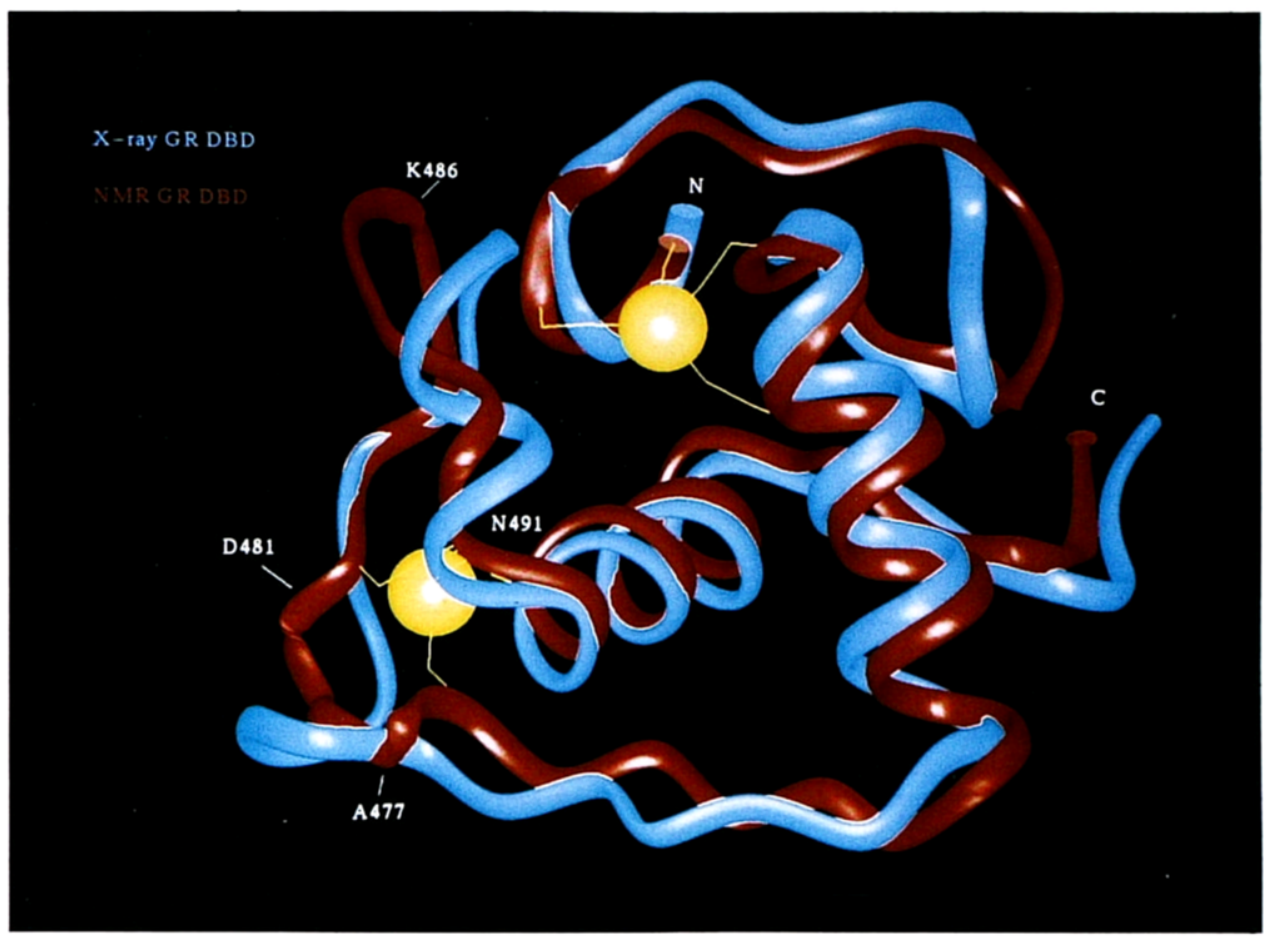

Figure 5. Ribbon representation of the, specifically bound, monomer crystal structure of GR DBD (Luisi et al., 1991) in blue, superimposed (residues Cys440 to Ser448, His451 to Gu469 and Pro493 to Glu508) on the average refined solution structure, colored red. The D-box (residues A477 to D481) and the disordered part of the second zinc finger (residues K486 to N491) are indicated.

domain shows several differences between the solution and crystal structure, which will now be discussed in detail.

\section{Disorder in second zinc finger and its implications for DNA binding}

Two disordered regions are found in the solution structure of the second zinc-coordinating domain; namely, the D-box (Ala477 to Asp481) and the region Lys486 to Asn491. Both regions form part of the dimerization interface in the homodimeric GR DBD-DNA complex. In solution the D-box is ill defined in both our refined structure and the refined structure reported by Baumann et al. (1993). However, in contrast to their findings we find no indication for the presence of a distorted helix in the second zinc finger, in spite of the fact that our structure is based on a somewhat larger number of NOE constraints (16.7 versus 13.7 constraints/ residue). In Table 4 a summary is given of the observed NOEs within this specific fragment of the two refined GR DBD structures (DBD 82 and DBD 93). From this Table it can be noticed that some specific helical contacts observed by Baumann $e t$ al. (1993) are absent in our 2D and 3D spectra. Only in two cases was this not unambiguous due to overlap. In all other cases the NOEs were genuinely absent, even at longer mixing times. Also indicated in Table 4 (by an asterisk) are the NOEs that would be expected based on the X-ray structure, because of proton-proton distances shorter than $4.5 \AA$ (Luisi et al., 1991).

Similar differences between NMR (free ER DBD without distorted helix) and X-ray structure were observed for the ER DBD (Schwabe et al., 1993a,b). It is noteworthy that even in the ER DBD-DNA complex only one of the dimeric complexes in the unit cell contained the distorted helix in the second zinc finger, while it is absent in the other complex (Schwabe et al., 1993b). NMR studies of RAR DBD (Knegtel et al., 1993) and RXR DBD (Lee et al., 1993) also revealed structures that are very similar to that of the GR DBD, without any indication of a distorted helix in the second zinc-coordinating domain.

Backbone dynamics of GR DBD (Berglund et al., 1992) did not show any evidence for rapid backbone motions within this specific region. However, ${ }^{15} \mathrm{~N}$ relaxation measurements register only fast, short time-scale dynamics, while motions involving the second zinc finger domain could take place on a longer time-scale inaccessible to current experimental approaches. Recently a thermodynamic analysis of protein-DNA interactions was reported by Spolar \& Record (1994). Based on the large heat capacity changes, Spolar \& Record (1994) inferred that for GR DBD folding occurs for approximately 18 residues per monomer upon DNA binding. This thermodynamic estimate would be in accordance with the folding of the whole second finger of GR DBD.

In the crystal structure a well-defined, hydrogenbonding network has been found in the tip of the first zinc-coordinating finger (His451-Ser448-Gly458), but this is not found in the refined structure presented here. A second hydrogen-bonding network is found in the second zinc-coordinating 
Table 4

\begin{tabular}{|c|c|c|c|c|c|c|c|c|}
\hline \multicolumn{9}{|c|}{ Observed NOEs in fragment D485 to N491 } \\
\hline & & D485 & K486 & I487 & R488 & R489 & $\mathrm{K} 490$ & $\mathrm{~N} 491$ \\
\hline$I, I+1$ & DBD82 & - $^{*}$ & $\mathrm{M}^{*}$ & $\mathrm{~S}^{*}$ & $\mathrm{~S}^{*}$ & $W^{*}$ & $\mathrm{~S}^{*}$ & 一 \\
\hline \multirow[t]{2}{*}{$d_{N N}$} & DBD93 & - & M & $S$ & S & $W$ & $S$ & W \\
\hline & DBD82 & $\mathrm{S}^{*}$ & $M^{*}$ & $M^{*}$ & $W^{*}$ & - $^{*}$ & $M^{*}$ & -* \\
\hline \multirow[t]{2}{*}{$d_{2 \mathrm{~N}}$} & DBD93 & $S$ & M & M & $W$ & - & M & M \\
\hline & DBD82 & $\mathrm{S}^{*}$ & $M^{*}$ & $M^{*}$ & $W^{*}$ & 一 $^{*}$ & $\mathrm{M}^{*}$ & $M^{*}$ \\
\hline$d_{B N}$ & DBD93 & $S$ & $\mathrm{M}$ & $S$ & - & - & $\mathrm{M}$ & - \\
\hline$l, l+2$ & DBD82 & 一 $^{*}$ & - & $\mathrm{M}^{*}$ & - $^{*}$ & - $^{*}$ & - $^{*}$ & 一 $^{*}$ \\
\hline \multirow{2}{*}{$d_{N N}$} & DBD93 & - & - & $W$ & - & - & - & 一 \\
\hline & DBD82 & $W^{*}$ & -* & $W^{*}$ & $W^{*}$ & 一 $^{*}$ & - $^{*}$ & —* \\
\hline \multirow{2}{*}{$d_{\mathrm{NN}}$} & DBD93 & - & - & - & $\#$ & - & - & - \\
\hline & DBD82 & $W^{*}$ & - & 一 $^{*}$ & - & - & - & - \\
\hline$d_{\mathrm{N}}$ & DBD93 & $W$ & - & - & - & - & - & - \\
\hline$I, I+3$ & DBD82 & $w$ & - & - & - & - & - & - \\
\hline \multirow[t]{2}{*}{$d_{N N}$} & DBD93 & $W$ & - & - & - & - & - & - \\
\hline & DBD82 & 一 $^{*}$ & $W^{*}$ & - $^{*}$ & $W^{*}$ & $W^{*}$ & - & - \\
\hline \multirow[t]{2}{*}{$d_{7 N}$} & DBD93 & - & - & $\#$ & - & - & - & - \\
\hline & DBD82 & 一 $^{*}$ & - & - $^{*}$ & $W^{*}$ & - & - & - \\
\hline$d_{2,1}$ & DBD93 & - & - & - & - & - & - & 一 \\
\hline \multirow{2}{*}{$\begin{array}{l}1, I+4 \\
d_{\mathrm{N} N}\end{array}$} & DBD82 & $W^{*}$ & - & - & 一 & - & - & - \\
\hline & DBD93 & $W$ & - & - & - & - & - & - \\
\hline \multicolumn{9}{|c|}{$\begin{array}{l}\text { Summary of the NOE connectivities observed within fragment D485-N491 of GR DBD in the structure (DBD } 82 \text { ) } \\
\text { reported by Baumann } \text { et } a l .(1993) \text { and the structure (DBD } 93 \text { ) presented here. S (strong), M (medium) and W (weak) } \\
\text { represent the relative NOE cross-peak intensities }(<2.7,<3.3 \text { and }<5 \AA \text {, respectively). A hash mark (\#) is indicated } \\
\text { where, due to overlap, assignment was not possible. In all other cases the cross-peaks were absent in the } 2 \mathrm{D} \text { and } 3 D \\
\text { spectra. The asterisk (*) indicates that this distance is shorter than } 4.5 \AA \text { in the X-ray structure. }\end{array}$} \\
\hline
\end{tabular}

domain of the crystal structure of the GR DBD DNA complex. Arg489, which is conserved in the superfamily and present in the disordered part, is involved in a four-way hydrogen-bonding network between Asp 445 and Ser 459 (in the first finger) to the backbone carbonyl group of Asn480 (D-box residue). This second network is not found in the solution structure. It seems reasonable, as discussed by Luisi et $a l$. (1991) and Schwabe et al. $(1993 a, b)$, that these hydrogen-bonding networks stabilize the protein in the complex with DNA. Absence of these networks could give rise to the ill-defined tip of the first finger and especially the disordering in the residues Ala477 to Asp481 and Lys486 to Asn491. Probably the formation of the hydrogen-bonding network upon dimerization as a consequence of DNA binding will stabilize the protein, forming the distorted helix and give rise to a conformational change of the D-box, as in the crystal structure.

\section{Conclusions}

The solution structure of the GR DBD (Cys 440 to Arg510) has been refined using relaxation matrix calculations constraints. Three cycles of IRMA refinement and a subsequent DINOSAUR refinement were performed using the initially reported NMR structure of GR DBD as a starting conformation.

Several secondary structure elements are present in the refined solution structures. A short stretch of anti-parallel $\beta$-sheet (Cys440-Leu 441 and Leu 455 to Cys457) is followed by a well-defined $\alpha$-helical region of residues Gly458 to Glu469. A second $\alpha$-helical region comprising Pro493 to Ala503 and a $\beta$-strain encompassing the residues Met505 to Leu507 follows a disordered region including the D-box (Ala477 to Asp481). The final refined structures have low $R$-factors, good stereochemical quality and low r.m.s.d. values for the best determined parts of the protein. Although analysis of backbone dynamics by ${ }^{15} \mathrm{~N}$ relaxation properties and the refined GR DBD reported by Baumann $c$ al . (1993) suggest that an $\alpha$-helical fold of the second finger is already present in the unbound state, we did not find indications for the presence of a distorted helix in spite of the fact that the presented structure is based on a larger number of NOE constraints. NMR and crystallographic studies of the homologous ER DBD in the unbound and bound state demonstrated that also in that case the second finger folds upon cooperative binding to DNA (Schwabe et al., 1993a,b). This model is also confirmed by thermodynamic analysis of induced secondary structure in GR DBD when bound to DNA (Spolar \& Record, 1994). Therefore we can conclude that, as our earlier work already suggested, DNA binding of GR DBD is accompanied by relatively large conformational changes in the region of the second zinc-finger.

\section{Materials and Methods}

\section{Sample preparation}

The protein fragment containing the GR DBD (KPARPICys440-Gly525]RL, numbering refers to the rat 
GR, including the residues of the T7 phage gene 10) was expressed as described (Freedman et al., 1988). Cells were grown on minimal medium with ${ }^{15} \mathrm{NH}_{4} \mathrm{Cl}$ as nitrogen source (Isotec Inc.). The protein was purified by elution from a CM Accell column (Waters) with a linear $50 \mathrm{mM}$ to $1 \mathrm{M} \mathrm{NaCl}$ gradient. Subsequently the protein was purified using a Superdex G75 column (Pharmacia). The protein was eluted with $20 \mathrm{mM}$ phosphate buffer (pH 7.0), $150 \mathrm{mM}$ $\mathrm{NaCl}, 1 \mathrm{mM}$ DTT. The purity of the protein was checked by gel electrophoresis. The purified protein was dialyzed and concentrated in an Amicon flow-cell against $95 \% \mathrm{H}_{2} \mathrm{O}$, $5 \%{ }^{2} \mathrm{H}_{2} \mathrm{O}$ for the $\mathrm{H}_{2} \mathrm{O}$ samples and $99.99 \%{ }^{2} \mathrm{H}_{2} \mathrm{O}$ for the ${ }^{2} \mathrm{H}_{2} \mathrm{O}$ samples containing $300 \mathrm{mM} \mathrm{NaCl}, 0.1 \mathrm{mM} \mathrm{NaN}_{3}$ and $1 \mathrm{mM}$ DTT to prevent oxidation of the cysteine residues. The final concentration was 3 to $4 \mathrm{mM}$ with a $\mathrm{pH}$ of 7.0.

\section{NMR-experiments}

${ }^{1} \mathrm{H}$ and ${ }^{15} \mathrm{~N}$ NMR spectra were recorded on Bruker AMX500 and AMX600 spectrometers, operating at $500 \mathrm{MHz}$ and $600 \mathrm{MHz}$, respectively. NOESY $(200 \mathrm{~ms}$ mixing time: Jeener et al., 1979) and heteronuclear (' $\mathrm{H}$ and ${ }^{15} \mathrm{~N}$ ) HSQC (Bodenhausen \& Ruben, 1980) spectra were recorded at $600 \mathrm{MHz}$ using TPPI quadrature detection in the $t_{1}$-domain (Marion \& Wüthrich, 1983). Three-dimensional heteronuclear NOESY-HSQC and TOCSY-HSQC spectra (Marion et al., 1989) were recorded at $600 \mathrm{MHz}$ at $297 \mathrm{~K}$ using 1024 complex data points in the acquisition domain and $200 t_{1}$ increments. $64 t_{2}$ increments, 16 scans/increment. A mixing time of $150 \mathrm{~ms}$ was used for the NOESY-HSQC and a $50 \mathrm{~ms}$ spin lock for the TOCSYHSQC.

NOE build-up curves were measured by recording five NOESY spectra in $\mathrm{H}_{2} \mathrm{O}$ and ${ }^{2} \mathrm{H}_{2} \mathrm{O}$ with mixing times of 30 , $60,90,120$ and $150 \mathrm{~ms}$. The spectra consisted of 512 increments of 64 scans each. The water resonance was suppressed by presaturation during the relaxation delay and during the mixing time. The NOESY spectra were recorded in one measuring session with a total measuring time of 60 hours. Both time domains were weighted with a sine-bell window function shifted by $\pi / 4$. All spectra were processed with the same parameters and were baseline-corrected in both dimensions. P.E.COSY (Müller, 1987; Bax \& Lerner, 1988) and DQF-COSY (Rance et al., 1983) spectra ( $800 t_{1}$ increments, 128 scans / increment and 4096 complex data points) were recorded using a $2 \mathrm{mM}$ sample of GR DBD at $300 \mathrm{~K}$, pH 6.7 at $500 \mathrm{MHz}$. All spectra were processed using the in-house TRITON software package.

\section{Distance constraints}

Distance constraints for distance geometry calculations were obtained from the NOE build-up curves using the $\mathrm{H}^{i}-\mathrm{H}^{\prime}$ cross-peaks of Tyr474 and Tyr497, corresponding to a distance of $2.45 \AA$, as a calibration. The NOE data were supplemented with 14 hydrogen-bond constraints from previously identified helices (Härd et al., 1990a), with upper and lower distance bounds between the amide proton of residue $(i+4)$ and the carbonyl oxygen atom of residue $(i)$ set to $2.3 \AA$ and $1.85 \AA$, respectively. Tetrahedral coordination of the zinc was imposed by adding 12 additional constraints by enforcing distance bounds of 3.83 $( \pm 0.02) \AA$ between sulfur atoms (Cys440, Cys443, Cys 457 and Cys 460 for the first finger, and Cys476, Cys482, Cys 492 and Cys495 for the second finger). These distances correspond to a tetrahedral coordination assuming a $\mathrm{Zn}-\mathrm{S}$ distance of $2.35 \AA$ as determined for the GR DBD by EXAFS spectroscopy (Freedman et al., 1988).

Stereospecific assignments for the $\gamma$-methyl groups of the four valine residues have been reported (Härd et al., $1990 a)$. Residues were judged to have a $\phi$ torsion angle of $-120( \pm 30)^{\circ}$ when its ${ }^{3} /{ }_{\mathrm{HN} \alpha}$ coupling constant was larger than $9 \mathrm{~Hz}$ (estimated from multiplets in a DQF-COSY). The $\phi$ angle could be restrained to $-120^{\circ}$ for ten residues. Stereospecific assignment for the $\beta$-protons of AMX spin systems were obtained from ${ }^{3} J_{\alpha \beta}$ coupling and intra-residue NOEs involving $\mathrm{H}^{\beta}, \mathrm{H}^{*}$ and $\mathrm{NH}$ protons (Hyberts et al., 1987 ), resulting in an additional set of 18 stereospecifically assigned residues, giving a total set of $22 \chi_{1}$ dihedral angle constraints. In the structure calculations, the $\chi_{1}$-angles were restrained to the corresponding values of $60^{\circ}, 180^{\circ}$ or $-60( \pm 30)^{\circ}$, which is shown in Table 5 . The NOEs involving the aromatic protons of Tyr452 on both sides of the ring could be assigned separately, since Tyr452 is rotating slowly on the NMR time-scale (Härd et al., 1990b). In cases where the stereospecific assignment was not known, the usual pseudoatom corrections were applied (Wüthrich, 1986). A total of 1186 distance constraints were used for the calculations corresponding to 435 intraresidue, 277 sequential, 170 medium-range $(2 \leqslant i-j \leqslant 4)$ and 304 long-range constraints $(i-j>4)$.

\section{Relaxation matrix calculations}

The strategy for the refinement of the three-dimensional structure of GR DBD from observed NOEs was similar to that recently used for the Arc repressor (Bonvin et al., 1994a). The refinement consists of two stages. In the first stage, accurate distances are obtained from IRMA, with inclusion of methyl rotation, aromatic ring flips and order parameters, which are used as constraints to generate and refine an ensemble of structures in a parallel protocol as proposed by Bonvin et al. (1993). In the second stage, the structures obtained from IRMA are refined directly against the experimental NOE data following a slow-cooling simulated annealing protocol using the DINOSAUR program (Bonvin et al., 1991).

IRMA calculations were performed for both $\mathrm{H}_{2} \mathrm{O}$ and ${ }^{2} \mathrm{H}_{2} \mathrm{O}$ data sets, with inclusion of fast methyl rotation and aromatic ring flips. The overall correlation time $\left(\tau_{c}\right)$ of $6.3 \mathrm{~ns}$ corresponds to a minimal $R$ factor. A leakage rate of $1.5 \mathrm{~s}^{-1}$, corresponding to the inverse average proton $T_{1}$, was added to the relaxation matrix diagonal. The NOE intensities of Cys457 $\mathrm{H}^{\beta 1}-\mathrm{H}^{\beta 2}$, Phe $464 \mathrm{H}^{\beta 1}-\mathrm{H}^{\beta 2}$, Ala467 $\mathrm{H}^{\alpha}-\mathrm{H}^{\beta}$, Tyr497 $\mathrm{H}^{-}-\mathrm{H}^{\circ}$ and Ala503 $\mathrm{H}^{\alpha}-\mathrm{H}^{\beta}$, were used for scaling of the experimental and theoretical NOE matrices.

In order to include the effect of fast local motions, generalized order parameters ( $S^{2}$; Lipari \& Szabo, 1982) were calculated from a free $M D$ simulation in water (Koning, 1990). A structure of GR DBD (Cys440 to Arg510) from the first IRMA-cycle was put in a box containing 2203 water molecules and periodic boundary conditions were used. One chlorine ion was added at the water position with the most positive electrostatic potential to compensate for.the net positive charge on the protein. Zinc coordination was maintained in the MD simulation by applying distance restraints between cysteine sulfur atoms and zinc ions, using a force constant of $45 \mathrm{~kJ} \mathrm{~mol}^{-1} \AA^{-2}$. To remove the unfavorable interactions, the system was first subjected to an energy minimization, with position restraining on the GR coordinates, with a force constant of $80 \mathrm{~kJ} \mathrm{~mol}^{-1} \AA^{-1}$. Initial velocities for the MD simulation were taken from a Maxwellian distribution at $300 \mathrm{~K}$. The MD simulation was carried out for $100 \mathrm{ps}$ with a time-step of $1.0 \mathrm{fs}$, keeping the temperature and 
Table 5

\begin{tabular}{|c|c|c|c|c|c|}
\hline \multicolumn{6}{|c|}{ Stereospecific assignment of the $\beta$-protons in AMX systems of DBD-93 } \\
\hline Residue & Rotamer & $\begin{array}{c}\mathrm{H}^{\rho \prime} \text { or } \\
\mathrm{H}^{\prime \prime} \text { for Val }\end{array}$ & $\begin{array}{c}\mathrm{H}^{\beta 2} \text { or } \\
\mathrm{H}^{22} \text { for Val }\end{array}$ & $\begin{array}{l}\text { Experimental } \\
\text { (deg.) }\end{array}$ & $\begin{array}{l}\text { NMR refined } \\
\text { (deg.) }\end{array}$ \\
\hline Cys440 & gt & 2.73 & 3.21 & 180 & $180 \pm 3$ \\
\hline Val442 & $\mathrm{g}^{+}$ & 0.89 & 1.16 & -60 & $-75 \pm 9$ \\
\hline Cys443 & gg & 2.80 & 1.96 & 60 & $68 \pm 4$ \\
\hline Asp445 & gt & 2.93 & 2.63 & 180 & $184 \pm 2$ \\
\hline Tyr452 & $\operatorname{tg}$ & 2.96 & 3.36 & -60 & $-51 \pm 10$ \\
\hline Val454 & $\mathrm{g}^{+}$ & 0.63 & 0.94 & -60 & $-65 \pm 16$ \\
\hline Cys457 & gg & 3.50 & 2.79 & 60 & $41 \pm 8$ \\
\hline Val462 & g' & 1.06 & 1.16 & -60 & $-69+14$ \\
\hline Phe463 & gt & 3.26 & 3.12 & 180 & $169+4$ \\
\hline Phe464 & gt & 3.62 & 3.02 & 180 & $172+3$ \\
\hline Val468 & $\mathrm{g}^{+}$ & 0.50 & 0.68 & -60 & $61 \pm 9$ \\
\hline Asn 473 & gt & 2.56 & 2.69 & 180 & $172 \pm 3$ \\
\hline Cys476 & gt & 2.79 & 2.81 & 180 & $185 \pm 6$ \\
\hline Asn 480 & gt & 2.97 & 2.51 & 180 & $189 \pm 7$ \\
\hline Asp481 & gg & 2.75 & 2.31 & 60 & $60 \pm 5$ \\
\hline Cys482 & gt & 2.97 & 2.51 & 180 & $165 \pm 12$ \\
\hline Asp485 & $\mathrm{gg}$ & 3.15 & 2.55 & 60 & $60 \pm 20$ \\
\hline Cys492 & $\mathrm{gt}$ & 2.90 & 2.70 & 180 & $174 \pm 9$ \\
\hline Cys495 & gt & 2.94 & 2.73 & 180 & $176 \pm 10$ \\
\hline Tyr497 & gt & 3.64 & 3.50 & 180 & $178 \pm 3$ \\
\hline Cys500 & $\operatorname{tg}$ & 3.06 & 2.97 & -60 & $-71 \pm 3$ \\
\hline Asn506 & gt & 2.80 & 2.74 & 180 & $180 \pm 4$ \\
\hline
\end{tabular}

' Rotamers gg, gt and tg denote gauche and trans position of $\beta_{1}$ and $\beta_{2}$ protons with respect to the $H^{x}$ protons. The rotamers $\mathrm{g}^{+}$denote the trans position of the $\gamma_{1}$-methyl group with respect to the $\mathrm{H}^{x}$ proton.

pressure in the system constant with relaxation times of 0.1 and $0.5 \mathrm{ps}$, respectively. Generalized order parameters were calculated from the last 55 ps as the average of the correlation functions between $t=12$ and $t=24 \mathrm{ps}$. These calculated order parameters were subsequently used in the second and third IRMA cycles and in the DINOSAUR refinement.

In the second step of the refinement, the experimental NOE intensities are directly used as structural constraints in a NOE restraining potential function as described (Bonzin et al., 1946b). A dynamic assignment procedure (Bonvin et al., 1994a,b) was applied to all NOEs involving diastereotopic protons that were not stereospecifically assigned and protons on aromatic rings that experience $180^{\circ}$ ring flips. The theoretical NOE intensities were calculated with the same parameters as in the IRMA calculations, except for the scaling; in DINOSAUR, an overall scaling factor was calculated with all peaks over all mixing times. For the NOE calculations, a spherical cut-off of $4.5 \AA$ around each proton pair defining a NOE is used for speeding up the calculations (Bonvin et al., 1994a).

\section{Structure refinement procedure}

A set of 50 structures was generated with the DGII program (Havel, 1991). Of the 50 structures, 47 wellconverged structures were further refined in parallel with a combination of REM followed by a 10 ps RMD and REM again, using the GROMOS force field (van Gunsteren \& Berendsen, 1987). REM was performed using a force constant of $40 \mathrm{~kJ} \mathrm{~mol}^{-1} \AA^{-2}$ for the harmonic distance restraining potential and $110 \mathrm{~kJ} \mathrm{~mol}^{-1}$ for the cosine dihedral angle restraining potential (de Vlieg et al., 1986). RMD calculations were performed in vacuo at a constant temperature of $300 \mathrm{~K}$ with a time-step of $2 \mathrm{fs}$. After the first IRMA cycle, a new set of constraints was obtained and the set of structures was further refined using REM and RMD. The best structure at that point was used as a starting structure in a free MD simulation in water to obtain order parameters. An average relaxation matrix was calculated from an ensemble of structures (according to $\left\langle r^{-6}\right\rangle$ averaging), with inclusion of the calculated order parameters and served as input for the second and third IRMA cycles. After the third IRMA cycle, convergence was obtained and 11 structures (selected in terms of $R$-factors and distance violations) were finally refined using DINOSAUR (Bonvin et al., 1991). For this we used a slow-cooling simulated annealing protocol as proposed by Bonvin et al. (1994b) consisting of three stages: 50 steps of steepest descent REM, 1.5 ps of slow-cooling MD with a time-step of $1 \mathrm{fs}$ from $800 \mathrm{~K}$ to $1 \mathrm{~K}$ with a cooling rate of $10 \mathrm{~K} / 19 \mathrm{fs}$ followed by 50 steps of steepest descent REM. $\mathrm{NOE}$, distances and dihedral angles constraints were applied in all three stages, with force constants of $400 \mathrm{~kJ} \mathrm{~mol}^{-1}, 2000 \mathrm{~kJ} \mathrm{~mol}^{-1} \mathrm{~nm}^{-1}$ and $110 \mathrm{~kJ} \mathrm{~mol}^{-1}$, respectively. In addition, the $\omega$ dihedral angles were restrained to $180^{\circ}$ during the slow-cooling MD with a force constant of $440 \mathrm{~kJ} \mathrm{~mol}^{-1}$.

The quality of experimental data and a model structure can be judged by comparing the calculated and experimental NOE intensities and can be described in an NMR residual factor ( $R$-factor; Rullmann et al., 1990; Borgias et al., 1990; Gonzales et al., 1991). We have used two definitions, the X-ray related definition by Gonzales et al. (1991) and the $Q^{1 / 6}$ factor as proposed by Bonzin et al. (1994a), which is less sensitive to errors in short distances and accounts for the asymmetry of the NOE function. In both definitions, the mixing times are used as weighting factors to emphasize NOEs measured at longer mixing times, which are expected to have a better signal to noise ratio. Final structures were also analyzed with respect to backbone dihedral angle conformation and hydrogen bonding. Dihedral angle order parameters $\left(S^{\psi^{\prime}}\right.$ and $S^{\phi}$; Hyberts et al., 1992) were calculated from the ensemble of 11 structures. Well-defined dihedral angles have order parameter values approaching unity. Hydrogen bonds were searched using as criteria a maximum distance of $2.5 \AA$ and anigle of $135^{\circ}$ between donor and acceptor. 


\section{Acknowledgements}

The authors thank Dr L. Freedman for the initial work on GR DBD, and we thank Dr P. B. Sigler for providing us with the crystal coordinates of the GR DBD/GRE complex. This work was supported by the Netherlands Organization for Chemical Research (SON). K. H. was supported by the Dutch Cancer Society.

\section{References}

Baumann, H., Paulsen, K., Kovács, H., Berglund, H., Wright, A. P. H., Gustafsson, J.-Å. \& Härd, T. (1993). Refined solution structure of the glucocorticoid receptor DNA-binding domain. Biochemistry, 32, 13463-13471.

Bax, A. \& Lerner, L. (1988). Measurement of ' $\mathrm{H}-{ }^{1} \mathrm{H}$ coupling constants in DNA fragments by 2D NMR. J. Magn. Reson. 79, 429-438.

Berglund, H., Kovács, H., Dahlman-Wright, K., Gustafsson, J.-A. \& Härd, T. (1992). Backbone dynamics of the glucocorticoid receptor DNA binding domain. Biochemistry, 31, 12000-12011.

Bodenhausen, G. \& Ruben, D. J. (1980). Natural abundance nitrogen-15 NMR by enhanced heteronuclear spectroscopy. Chem. Phys. Letters, 69, 185-189.

Boelens, R., Koning, T. M. G. \& Kaptein, R. (1988). Determination of biomolecular structures from proton-proton NOEs using a relaxation matrix approach. J. Mol. Struct. 173, 299-311.

Boelens, R., Koning, T. M. G., van der Marel, G. A., van Boom, J. M. \& Kaptein, R. (1989). Iterative procedure for structure determination from proton-proton NOEs using a full relaxation matrix approach. Application to a DNA octamer. J. Magn. Reson. 82, 290-308.

Bonvin, A. M. J. J., Boelens, R. \& Kaptein, R. (1991). Direct NOE refinement of biomolecular structures using 2D NMR data. J. Biomol. NMR, 1, 305-309.

Bonvin, A. M. J. J., Rullmann, J. A. C., Lamerichs, R. M. J. N., Boelens, R. \& Kaptein, R. (1993). Ensemble iterative relaxation matrix approach: a new NMR refinement protocol applied to the solution structure of crambin. Proteins: Struct. Funct. Genet. 15, $385-400$.

Bonvin, A. M. J. J., Vis, H., Breg, J. N., Burgering, M. J. M., Boelens, R. \& Kaptein, R. (1994a). NMR solution structure of the Arc repressor using relaxation matrix calculations. J. Mol. Biol. 236, 328-341.

Bonvin, A. M. J. J., Boelens, R. \& Kaptein, R. (1994b). Direct NOE refinement of crambin from 2D NMR data using a slow cooling simulated annealing. Biopolymers, 34, 39-50.

Borgias, B. A., Cochin, M., Kerwood, D. J. \& James, T. L. (1990). Relaxation matrix analysis of 2D NMR data. Prog. NMR Spectrosc. 22, 83-100.

de Vlieg, J., Boelens, R., Scheek, R. M., Kaptein, R. \& van Gunsteren, W. F. (1986). Restrained molecular dynamics procedure for protein tertiary structure determination from NMR data: a Lac repressor headpiece structure based on information on J-couplings and presence of NOEs. Isr. J. Chem. 27, $181-188$.

Evans, R. M. (1988). The steroid and thyroid hormone receptor superfamily. Science, 240, 889-894.

Freedman, L. P., Luisi, B. F., Korszum, Z. R., Basavappa, R., Sigler, P. B. \& Yamamoto, K. R. (1988). The function and structure of the metal coordination sites within the glucocorticoid receptor DNA binding domain. Nature (London), 344, 543-546.

Gonzalez, C., Rullmann, J. A. C., Bonzin, A. M. J. J., Boelens, R. \& Kaptein, R. (1991). Toward an NMR R-factor. J. Magn. Reson. 91, 659-664.

Ham, J. \& Parker, M. G. (1989). Regulation of gene expression by nuclear hormone receptors. Curr. Opin. Cell Biol. 1, 503-511.

Härd, T., Kellenbach, E., Boelens, R., Dahlman, K., Carlstedt-Duke, J., Freedman, L., Maler, B., Hyde, E., Gustafsson, J.-Å., Yamamoto, K. \& Kaptein, R. (1990a). 'H NMR studies of the glucocorticoid receptor DNA-binding domain: sequential assignments and identification of secondary structure elements. Biochemistry, 29, 9015-9023.

Härd, T., Kellenbach, E., Boelens, R., Maler, B., Dahlman, K., Freedman, L., Carlstedt-Duke, J., Yamamoto, K., Gustafsson, J.-A. \& Kaptein, R. (1990b). Solution structure of the glucocorticoid receptor DNA-binding domain. Science, 249, 157-160.

Havel, T. F. (1991). An evaluation of computational strategies for use in the determination of protein structures from distance constraints obtained by nuclear magnetic resonances. Prog. Biophys. Mol. Biol. 56, 43-78.

Hyberts, S. G., Märki, W. \& Wagner, G. (1987). Stereospecific assignments of side-chain protons and characterisation of torsion angles in eglin C. Eur. J. Biochem. 164, 625-635.

Hyberts, S. G., Goldberg, M. S., Havel, T. F. \& Wagner, G. (1992). The solution structure of eglin C. based on the measurements of many NOEs and coupling constants and its comparison with X-ray structures. Protein Sci. 1, 736-751.

Jeener, J., Meier, B. H., Bachmann, P. \& Ernst, R. R. (1979). Investigation of exchange processes by two-dimensional NMR spectroscopy. J. Chem. Phys. 71, 4546-4553.

Kabsch, W. \& Sander, C. (1983). Dictionary of protein secondary structure: pattern recognition of hydrogen bonded and geometrical features. Biopolymers, 22, 2577-2637.

Kaptein, R., Zuiderweg, E. R. P., Scheek, R. M., Boelens, R. \& van Gunsteren, W. F. (1985). A protein structure from nuclear magnetic resonance data. Lac repressor headpiece. J. Mol. Biol. 182, 179-182.

Knegtel, R. M. A., Katahira, M., Schilthuis, J. G., Bonvin, A. M. J. J., Boelens, R., Eib, D., van der Saag, P. T. \& Kaptein, R. (1993). The solution structure of the human retinoic acid receptor- $\beta$ DNA-binding domain. J. Biomol. NMR, 3, 1-17.

Koning, T. M. G. (1990). IRMA: iterative relaxation matrix approach for NMR structure determination. Application to DNA fragments, $\mathrm{PhD}$ thesis, Utrecht University.

Koning, T. M. G., Boelens, R. \& Kaptein, R. (1990). Calculation of the nuclear Overhauser effect and the determination of proton-proton distances in the presence of internal motions. J. Magn. Reson. 90, 111-123.

Koning, T. M. G., Boelens, R., van der Marel, G. A., van Boom, J. A. \& Kaptein, R. (1991). Structure determination of a DNA octamer in solution by NMR spectroscopy. Effect of fast local motions. Biochemistry, 30, 3787-3797.

Lee, M. S., Kliewer, S. A., Provencal, J., Wright, P. E. \& Evans, R. (1993). Structure of the retinoid $X$ receptor $\alpha$ domain: a helix required for homodimeric DNA binding. Science, 260, 1117-1121. 
Lipari, G. \& Szabo, A. (1982). Model-free approach to the interpretation of nuclear magnetic resonance relaxation in macromolecules. 1. Theory and range of validity. 2. Analysis of experimental results. J. Amer. Chem. Soc. 104, 4546-4570.

Luisi, B. F., Xu, W. X., Otwinowski, Z., Freedman, L. P., Yamamoto, K. R. \& Sigler, P. B. (1991). Crystallographic analysis of the interaction of the glucocorticoid receptor with DNA. Nature (London), 352, 572-575.

Marion, D. \& Wüthrich, K. (1983). Application of phase sensitive two-dimensional correlated spectroscopy (COSY) for measurement of ${ }^{1} \mathrm{H}-{ }^{1} \mathrm{H}$ spin-spin coupling constants in proteins. Biochem. Biophys. Res. Commun. 113, 967-974.

Marion, D., Driscoll, P. C., Kay, L. E., Wringfield, P. T., Bax, A., Gronenborn, A. M. \& Clore, G. M. (1989). Overcoming the overlap problem in the assignment of ' $\mathrm{H}$ NMR spectra of larger proteins by use of 3D heteronuclear ${ }^{1} \mathrm{H}-{ }^{15} \mathrm{~N}$ HartmannHahn-multiple quantum coherence and nuclear Overhauser multiple quantum coherence spectroscopy: application to interleukine $1 \beta$. Biochemistry, 28, 6150-6156.

Morris, A. L., MacArthur, M. W., Hutchinson, E. G. \& Thornton, J. M. (1992). Stereochemical quality of protein structure coordinates Proteins: Struct. Funct. Genet. 12, 345-364.

Müller, L. (1987). P.E.COSY, a simple alternative to E.COSY. J. Mngn. Reson. 72, 191-196.

Rance, M., Sorenson, O. W., Bodenhausen, G., Wagner, G., Ernst, R. R. \& Wuthrich, K. (1983). Improved spectral resolution in COSY 'H NMR spectra of proteins via double quantum filtering. Biochem. Biophys. Res. Commun. 117, 479-485.

Rullmann, J. A. C., Lamerichs, R. M. J. N., Gonazalez, C., Koning, T. M. G., Boelens, R. \& Kaptein, R. (1990). Structure determination from NMR using a relaxation matrix approach; application to the solution structure of Crambin. In Rivail J-L (Ed) Modelling of Molecular Structures and Properties, Studies in Physical and Theoretical Chemistry (Rivail, J.-L., ed.); vol.71, pp. 703-710, Elsevier, Amsterdam.

Schwabe, J. W. R., Neuhaus, D. \& Rhodes, D. (1990). Solution structure of the DNA binding domain of the oestrogen receptor. Nature (London), 348, 458-461.

Schwabe, J. W. R., Chapman, L., Finch, J. T., Rhodes, D. \& Neuhaus, D. (1993a). DNA recognition by the oestrogen receptor: from solution to the crystal. Structure, 1, 187-204.

Schwabe, J. W. R., Chapman, L., Finch, J. T. \& Rhodes, D. $(1993 b)$. The crystal structure of the estrogen receptor DNA-binding domain bound to DNA: how receptors discriminate between their response elements. Cell, 348, 567-578.

Spolar, R. \& Record, T., Jr (1994). Coupling of local folding to site-specific binding of protein to DNA. Science, 263, 777-784.

van Gunsteren, W. F. \& Berendsen, H. J. C. (1987). Groningen Molecular Simulation (GROMOS) Library Manual. Biomos BV, Nijenborgh 16, 9747 AG Groningen, The Netherlands.

Wüthrich, K. (1986). NMR of Proteins and Nucleic Acids, John Wiley and Sons, New York.

Edited by P. E. Wright

(Received 13 October 1994; accepted 9 January 1995) 